\title{
A GEOMETRIC CHARACTERIZATION OF GLEASON PARTS
}

\author{
H. S. BEAR $^{1}$
}

1. Introduction. In [3] Gleason introduced an equivalence relation on the maximal ideal space $S_{A}$ of a function algebra $A$, which divides $S_{A}$ into equivalence classes called "parts", or "Gleason parts". The decomposition of $S_{A}$ into these parts is an important step in the attempt to find whatever analytic structure $S_{A}$ may have (cf. Wermer's elegant result in [7]). Among other properties of parts, Gleason showed that any two points in the same part of a Dirichlet algebra have representing measures on the Silov boundary which are mutually absolutely continuous. Bishop [1] has recently given a simple proof that this fact holds for any function algebra. Motivated by the ideas of Bishop's proof, we wish to extend the notion of Gleason part to an arbitrary linear space $B$ of continuous real functions on a compact space $X$. The parts of $X$ determined by $B$ are then subsets of $X$ on which a Harnack inequality holds, in much the same way that the Gleason parts for an algebra on $X$ are subsets of $X$ on which a type of Schwarz lemma holds. The equivalence relation we introduce is a generalization of Gleason's, in the sense that if $A$ is an algebra on $X$, and $B$ is the space of real parts, then the classes of $X$ induced by $B$ coincide with the Gleason parts. We also introduce a metric on the parts of $X$ induced by $B$, and show that, for the case $B=\operatorname{Re} A$, it is equivalent to the norm metric studied by Gleason. Finally, the parts are characterized geometrically as the minimal faces of a certain compact convex subset of the dual space.

The author would like to express his appreciation to Errett Bishop for a pre-publication exposition of [1], and to $\mathrm{H}$. H. Corson for several helpful conversations concerning this paper.

2. The parts of $X$ induced by $B$. Let $X$ be a compact Hausdorff space, and let $C_{r}(X)$ (respectively, $\left.C_{c}(X)\right)$ be the space of all continuous real-valued (respectively, complex-valued) functions on $X$, with the uniform norm. We consider a linear subspace $B$ of $C_{r}(X)$, containing the constant functions, and separating the points of $X$.

For any two points $x, y \in X$ write $x \sim y(a)$ if and only if

$$
1 / a<u(x) / u(y)<a
$$

Presented to the Society, April 18, 1964; received by the editors December 24, 1963.

1 This research has been supported in part by Grant NSF-GP-216 of the National Science Foundation. 
for all strictly positive functions $u \in B$. The condition (1) can be viewed as an abstract form of Harnack's inequality for positive harmonic functions. If $x \sim y(a)$ then, clearly, $a>1$. We write $x \sim y$ if and only if $x \sim y(a)$ for some $a$. It is easy to check that $\sim$ is an equivalence relation on $X$ and we state the relevant facts in the following lemma.

LemMA 1. (i) $x \sim x$ (a) for all $a>1$; (ii) $x \sim y$ (a) implies $y \sim x$ (a); (iii) $x \sim y(a)$ and $y \sim z(b)$ implies $x \sim z(a b)$.

The equivalence classes of $X$ under $\sim$ will be called the "parts", or "Gleason parts" of $X$ induced by $B$. For $x$ and $y$ in the same part, we define

$$
R(x, y)=\inf \{a: x \sim y(a)\} .
$$

The following is immediate from Lemma 1 and this definition.

Lemma 2. If $x, y, z$ are in the same part of $X$, then (i) $R(x, y)$ $\geqq 1$ and $R(x, y)=1$ if and only if $x=y$; (ii) $R(x, y)=R(y, x)$; (iii) $R(x, y) R(y, z) \geqq R(x, z)$.

Let $D$ be the function on all pairs $(x, y)$ of points in the same part defined by

$$
D(x, y)=\log R(x, y) .
$$

Lemma 3. $D$ is a metric on each part of $X$.

3. Relationship with the Gleason parts of an algebra. Let $A$ be a closed subalgebra of $C_{c}(X)$, and assume that $A$ contains the constant functions and separates the points of $X$. We will say " $A$ is an algebra on $X^{\prime}$ if these conditions hold. If $A^{*}$ is given the $w^{*}$ topology, then $X$ is homeomorphic to the subset of $A^{*}$ consisting of the evaluation functionals $e_{x}$ defined by $e_{x}(f)=f(x)$ for all $f \in A$. We can transfer the norm-metric of $A^{*}$ to $X$ via this identification, and we write, for $x, y \in X$,

(4) $G(x, y)=\left\|e_{x}-e_{y}\right\|=\sup \{|f(x)-f(y)|: f \in A,\|f\|<1\}$.

Clearly $G$ is a metric, and $G(x, y) \leqq 2$ for all $x, y$.

Theorem 4 (Gleason [3]). If $G(x, y)<2$ and $G(y, z)<2$, then $G(x, z)<2$.

Theorem 4 implies that the relation $G(x, y)<2$ is an equivalence relation on $X$, which we denote $x \approx y$. We will need the following fact, which follows from the properties of conformal mappings of the unit 
disc, applied to the range of functions in $A$. The points $x, y$ are not equivalent ( $x \approx y$ fails, or $G(x, y)=2$ ) if and only if for some $s<1$ and every $\epsilon>0$, there is $f \in A,\|f\|<1$, with $|f(x)| \leqq s$ and $|f(y)|>1-\epsilon$. The following theorem shows that the relation $\sim$ does specialize to the Gleason relation $\approx$ in the case of a function algebra. The basic idea of this theorem is due to Bishop [1] who showed in somewhat different language that $x \approx y$ implies $x \sim y$.

Theorem 5. If $A$ is an algebra on $X$ and $B=\operatorname{Re} A$, then $G(x, y)<2$ if and only if $1 / a<u(x) / u(y)<a$ for some $a$ and all positive $u \in B$.

We can state the theorem briefly: $x \sim y$ if and only if $x \approx y$.

Proof. Assume that condition (1) does not hold; that is, $x \sim y$ fails. If, to be specific, $u(x) / u(y)$ is not bounded away from zero, then there is a strictly positive $u \in B$ such that $u(x)<\epsilon$ and $u(y)=1$. If $u+i v \in A$ and $f=e^{-u-i v}$, then $f \in A,|f|=e^{-u}<1,|f(y)|=e^{-1}$, and $|f(x)|>e^{-\epsilon}$. Thus there are functions $f$ in $A$ of norm $\leqq 1$ which map $x$ arbitrarily close to the boundary of the disc, while $|f(y)|$ is bounded away from 1. This shows that $G(x, y)=2$ if $x \sim y$ fails.

Now assume $G(x, y)=2$. Given $\epsilon>0$ there is therefore $f=u+i v \in A$ such that $\|f\|<1$ and $u(x)<-1+\epsilon, u(y)>1-\epsilon$. If $u_{0}=u+1$, then $u_{0} \in B, u_{0}>0$ on $X$, and $u_{0}(x) / u_{0}(y)<\epsilon /(2-\epsilon)$. Hence $x \sim y$ fails if $x \approx y$ fails.

Theorem 6. If $A$ is an algebra on $X$, and $B=\operatorname{Re} A$, then the metric $G$ of (4) is equivalent to the metric $D$ of (3) on each part of $X$.

Proof. Fix a part of $X$, and consider only points $x, y$ in this part. We show that if $G(x, y) \geqq r>0$, then $D(x, y) \geqq \log (1+r / 2)$, and secondly, that if $D(x, y) \geqq r>0$, then $G(x, y) \geqq e^{-1}-e^{-1-r}$.

Assume first that $G(x, y) \geqq r>0$. For any $\epsilon>0$ there is $f \in A,\|f\|<1$, such that $|f(x)-f(y)|>r-\epsilon$. We can assume, by rotating the graph of $f$ if necessary, that $f=u+i v$ and $u(x)-u(y)>r-\epsilon$. If $u_{0}=u+1$, then $0<u_{0}<2$ on $X, u_{0}(x)-u_{0}(y)>r-e$, and

$$
u_{0}(x) / u_{0}(y)>\left(u_{0}(y)+r-\epsilon\right) / u_{0}(y)>1+(r-\epsilon) / 2 .
$$

Hence $R(x, y) \geqq 1+r / 2$, and $D(x, y) \geqq \log (1+r / 2)$.

Now assume that $D(x, y)=\log R(x, y) \geqq r>0$. Since $R(x, y) \geqq e^{r}$ $>1+r$, there is a positive function $u \in B$ with $u(x) / u(y)>1+r$ (or $u(y) / u(x)>1+r$ with a similar proof). We normalize $u$ so that $u(y)=1$ and $u(x)>1+r$. Let $u+i v \in A$, and $f=e^{-u-i v}$. Then $f \in A,\|f\|<1$, and

$$
|f(x)-f(y)| \geqq|f(y)|-|f(x)|=e^{-u(y)}-e^{-u(x)}>e^{-1}-e^{-1-r} \text {. }
$$

Coroliary. Each $f \in A$ is continuous on each part of $X$ with the metric $G$ or $D$. 
Proof. This is obvious for the metric $G$, since the norm topology in $A^{*}$ is stronger than the $w^{*}$ topology, which coincides on the parts with the given topology. Hence the functions are also continuous in the equivalent metric $D$.

4. Geometric characterization of parts. Our geometric characterization of the Gleason parts for a subspace $B$ of $C_{r}(X)$ (and hence of a subalgebra $A$ of $\left.C_{c}(X)\right)$ makes use of the representation of $B$ as the weak dual of $B^{*}$. Give $B^{*}$ the $w^{*}$ topology, and, for each $u \in B$, define $\bar{u}$ on $B^{*}$ by $\bar{u}(F)=F(u)$ for all $F \in B^{*}$. The space $\bar{B}$ of all such functions $\bar{u}$ is isomorphic to $B$, and is the space of all $w^{*}$-continuous linear functionals on $B^{*}([2$, p. 18$]$ or $[4$, Problem W, p. 108]). If $X^{e}=\left\{e_{x}: x \in X\right\} \subset B^{*}$, then $X^{e}$ is homeomorphic to $X$ and $\bar{B} \mid X^{e}$ is a copy of $B$ on $X$, since $\bar{u}\left(e_{x}\right)=e_{x}(u)=u(x)$. Let $T_{B}$ denote the closed convex hull of $X^{e}$ in $B^{*}$. The following theorem can be derived as a consequence of the Riesz representation theorem and the fact that linear combinations of point masses are $w^{*}$ dense in the space of all measures. We include a simple proof which does not involve any notion of measure or integration.

TheOREM 7. $T_{B}=\left\langle X^{\bullet}\right\rangle=\left\{F \in B^{*}: F(1)=\|F\|=1\right\}$.

Proof. Since $B^{*}$ is a locally convex space, and $\bar{B}$ is its dual, the closed convex hull of $X^{\bullet}$ can be written [2, Corollary 2, p. 22]

$$
\begin{aligned}
T_{B}=\left\langle X^{e}\right\rangle & =\left\{F \in B^{*}: \bar{u}(F) \leqq \max \bar{u}\left[X^{e}\right], \quad \text { all } u \in B\right\} \\
& =\left\{F \in B^{*}: F(u) \leqq \max u, \quad \text { all } u \in B\right\} .
\end{aligned}
$$

For $F \in T_{B}$ we have, from (5), $F(u) \leqq \max u \leqq\|u\|$ and $-F(u)$ $\leqq F(-u) \leqq \max (-u) \leqq\|u\|$. Hence $-\|u\| \leqq F(u) \leqq\|u\|$, and $\|F\| \leqq 1$. In addition, $-F(1)=F(-1) \leqq \max (-1)=-1$, so $F(1)=1$, and $\|F\|=F(1)=1$ if $F \in T_{B}$. Now suppose that $\|F\|=F(1)=1$. If $F \notin T_{B}$, then $F\left(u_{0}\right)>\max u_{0}$ for some $u_{0} \in B$. For sufficiently large $n, u_{0}+n$ $\geqq 0$, and $\left\|n+u_{0}\right\|=n+\max u_{0}$. Since $F(1)=1, F\left(n+u_{0}\right)=n+F\left(u_{0}\right)$ $>\left\|n+u_{0}\right\|$, which contradicts the assumption $\|F\|=1$.

The set $T_{B}$, which we will call the carrier of $B$, is a compact convex set in the locally convex space $B^{*}, w^{*}$. It is the largest compact space $K$ containing $X$ such that $B$ can be extended to $K$ to be isomorphic and isometric with a separating subspace of $C_{r}(K)$ containing the constants. In this sense, the carrier $T_{B}$ plays somewhat the role for a linear space $B$ that the spectrum $S_{A}$ does for an algebra $A$. If $A$ is an algebra on $X$, and $B=\operatorname{Re} A$, then $S_{A}$ can be considered a subset of $T_{B}$, since the homomorphisms of $A$ are positive linear functionals of norm one (cf. [6, p. 58]). We can therefore consider $A$ as linearly isomorphic 
and isometric to a linear space $\bar{A}$ of complex linear functionals on $T_{B}$, with $\bar{A} \mid S_{A}$ being algebraically isomorphic to $A$.

To simplify the notation, and without loss of generality, we will henceforth regard $X$ as a compact subset of a locally convex space, $B^{*}$, with compact closed convex hull $T_{B}$, and $B$ as the space of all continuous linear functionals on $B^{*}$, usually restricted to $X$ or to $T_{B}$. We use $x, y, z$ for points of $X$, or $T_{B}$, or functionals in $B^{*}$.

We will call a subset $E$ of a convex set $K$ a face of $K$ if and only if $E$ is convex and $(a, b) \subset E$ whenever the segment $(a, b)$ intersects $E$ and $(a, b) \subset K$. Note that a face differs from the "extremal set" of $[2$, p. 78] and the "support set" of $[5$, p. 130] in that a face should not contain all end points of segments $[a, b]$ which intersect it. A face is "open" in this sense. A nonempty intersection of faces is obviously a face. We will let $S(x)$ denote the smallest face of $T_{B}$ containing $x$; i.e., $S(x)$ is the intersection of all faces of $T_{B}$ containing $x$.

LEMмA 8. Either $x$ is an extreme point of $T_{B}$, or $S(x)$ is the union of all open segments $\left(a^{\prime}, a^{\prime \prime}\right)$ in $T_{B}$ which contain $x$.

Proof. Suppose $x$ is not an extreme point of $T_{B}$. Clearly $S(x)$ must contain all open segments $\left(a^{\prime}, a^{\prime \prime}\right)$ such that $x \in\left(a^{\prime}, a^{\prime \prime}\right) \subset T_{B}$, so it is sufficient to show that the union $E$ of such segments is a face. If $a, b \in E$, then $a \in\left(a^{\prime}, a^{\prime \prime}\right) \subset T_{B}$, and $b \in\left(b^{\prime}, b^{\prime \prime}\right) \subset T_{B}$, and $x$ is in both segments. The points $a, a^{\prime}, a^{\prime \prime}, b, b^{\prime}, b^{\prime \prime}, x$ all lie in a two-dimensional plane in $T_{B}$, and it is easy to see from the plane geometry that each point of $(a, b)$ is in an open segment of $T_{B}$ containing $x$. Thus $E$ is convex. It similarly is clear from the two-dimensional picture that if $y \in E$ and $y \in(a, b) \subset T_{B}$, that $(a, b) \subset E$.

It follows from the definition of a face that if $y \in S(x)$, then $S(y) \subset S(x)$. On the other hand, from Lemma 8 we have that $y \in S(x)$ if and only if $x \in S(y)$. Thus $y \in S(x)$ if and only if $S(x)=S(y)$.

We call attention to the fact that Lemma 8 shows that any convex set $K$ decomposes into disjoint convex sets which are its minimal faces, and that each face $F$ (other than an extreme point) can be characterized as the union of all open segments in $K$ through any point of $F$.

THEOREM 9. If $x$ and $y$ are points of $T_{B}$, then $x \sim y$ if and only if $S(x)=S(y)$. That is, the Gleason parts of $T_{B}$ are its minimal faces, and the Gleason parts of $X$ are the intersections of $X$ (properly $\left.X^{e}\right)$ with the minimal faces of $T_{B}$.

Proof. Assume that $S(x) \neq S(y)$, and to be definite assume that $x$ is not interior to any segment in $T_{B}$ containing $y$. Let $z_{n}$ be the point of $B^{*}$ such that 


$$
x=\frac{n-1}{n} z_{n}+\frac{1}{n} y .
$$

Then $z_{n} \notin T_{B}$ by hypothesis. However, $z_{n}$ is in the hyperplane $\left\{F \in B^{*}: F(1)=1\right\}$, since $x(1)=1$ and $y(1)=1$. Let $u \in B$, and $u\left(z_{n}\right)$ <inf $u\left[T_{B}\right]=a$ (such $u$ exists by [2, Theorem 5, p. 22]). If $u_{0}=u-a$ $\in B$, then $u_{0}\left(z_{n}\right)=u\left(z_{n}\right)-a<0=\inf u_{0}\left[T_{B}\right]$. From (6) we have $u_{0}(x)$ $<(1 / n) u_{0}(y)$. Since $n$ is arbitrary, $x \sim y$ fails if $S(x) \neq S(y)$. Now assume that $x$ and $y$ are not in the same part, and, to be definite, that $u(x) / u(y)$ is not bounded away from zero for $u>0$ on $T_{B}$. Then there is no $z \in T_{B}$ such that $x \in(y, z)$, for, if $x=\lambda y+(1-\lambda) z$, and $z \in T_{B}$, then $u(x) / u(y) \geqq \lambda$ for all positive $u \in B$. From the preceding lemma we conclude that $x \in S(y)$ and hence that $S(x) \neq S(y)$.

Let us make explicit what the theorem above says for a function algebra $A$. Suppose that $A$ is an algebra on $X$, which may be assumed to be the Silov boundary of $A$, the whole maximal ideal space $S_{A}$, or any closed set in between. If $B=\operatorname{Re} A$, then $X$ is (homeomorphic to) a subset of $T_{B}$, and $A$ can be considered a family of complex linear functionals on $T_{B}$, with $A$ closed under multiplication when restricted to $X$. The Gleason parts of $X$ with respect to $A$ are the intersections of $X$ with the minimal faces of $T_{B}$. Using the characterization of $T_{B}$ in Theorem 7 , we can alternatively say that each part of $S_{A}$ consists of the multiplicative functionals in one of the minimal faces of the set $\left\{F \in B^{*}: F(1)=\|F\|=1\right\}$.

\section{REFERENCES}

1. Errett Bishop, Representing measures for points in a uniform algebra, Bull. Amer. Math. Soc. 70 (1964), 121-122.

2. Mahlon M. Day, Normed linear spaces, Springer, Berlin, 1958.

3. A. M. Gleason, Function algebras, Seminars on Analytic Functions, Vol. 2, Institute for Advanced Study, Princeton, N. J., 1957.

4. J. L. Kelley, General topology, Van Nostrand, New York, 1955.

5. J. L. Kelley, Isaac Namioka, et al., Linear topological spaces, Van Nostrand, New York, 1963.

6. John Wermer, Banach algebras and analytic functions, Advances in Math. 1 (1961), 51-102.

7. —, Dirichlet algebras, Duke Math. J. 27 (1960), 373-382.

University of California, Santa Barbara 